\title{
Investigation of the Contraction Ratio of Transversus Abdominis and Internal Oblique Muscles during Lumbopelvic Stability Test
}

\author{
P. Sitilertpisan ${ }^{1}$, L. Joseph², A. Paungmali', U. Pirunsan ${ }^{1}$, T. Chunchai ${ }^{3}$ \\ 1 Department of Physical Therapy, Faculty of Associated Medical Sciences, Chiang Mai University, Chiangmai, Thailand \\ 2 School of Health Science, University of Brighton, East Sussex, United Kingdom \\ 3 Department of Physiology, Faculty of Medicine, Chiang Mai University, Chiangmai, Thailand
}

\author{
CORRESPONDING AUTHOR: \\ Patraporn Sitilertpisan \\ Department of Physical Therapy \\ Faculty of Associated Medical Sciences \\ Chiang Mai University \\ Chiang Mai, Thailand \\ Phone: +66 0-5394-9243 \\ E-mail: patraporn.s@cmu.ac.th
}

DOI:

10.32098/mltj.01.2020.10

LEVEL OF EVIDENCE: $2 B$

\begin{abstract}
SUMMARY
Background. The clinicians administer lumbopelvic stability test (LPST) to evaluate core stability of lumbar spine and TrA muscle function. While the reduced thickness and contraction ratio of the TrA was well documented among people with nonspecific low back pain (NSLBP), the direct morphological changes of TrA during the LPST had not been reported so far.

Purpose. To determine and compare the contraction ratio of deep abdominal muscles ( $\mathrm{TrA}$ and IO) during the 7 progressive stages of the LPST in healthy individuals.

Methods. Thirty healthy volunteers ( 15 males and 15 females) aged $21.83 \pm 0.46$ years participated in an experimental study. The TrA and IO thickness was assessed by ultrasound imaging (USI) TOSHIBA, Famio8, SSA-530A) at the right side in mid axillary line between $12^{\text {th }}$ rib and iliac crease. Images were taken for 2 trials during each level of LPST (7 levels) as measured by the pressure biofeedback unit (PBU). The thickness of the $\mathrm{Tr} A$ and IO muscles were measured by Image J program (Image J®, NIH, USA) and the contraction ratio was calculated. A one way repeated measure ANOVA with post hoc analysis using the Bonferroni test at $\mathrm{p}<0.05$ was used to analyse the data.

Results. The contraction ratio of TrA showed a decreasing trend as the LPST was progressed to the 7 levels. Post hoc analysis showed a significant reduction in the contraction ratio of the TrA muscle in the $7^{\text {th }}$ level of the LPST $(F=14.53, p=0.001)$. However, the contraction ratio of IO remains unchanged during all the 7 levels of LPST.

Conclusions. The TrA muscle responded by reduced contraction ratio across the 7 levels of the LPST as measured by the PBU and USI. Further studies are warranted to compare the adaptation of the TrA muscle across 7 levels of the LPST among patients with NSLBP.
\end{abstract}

KEY WORDS

Low back pain; musculoskeletal; physiotherapy; rebabilitation; transversus abdominis; ultrasound imaging

\section{INTRODUCTION}

Approximately $90 \%$ of the pain originating from the lumbar spine are classified as non-specific low back pain (NSLBP) (1). The mechanical overuse and dysfunction of the spine and the surrounding structures such as core muscles were proposed as one of the contributing factors for NSLBP (1). An impaired function of the deep core muscles such as transversus abdominis ( $\operatorname{Tr} A)$ and internal oblique (IO) was reported to affect stability, robustness and movement control of the lumbar spine leading to recurrent low back pain (2). TrA and IO as deep abdominal muscles particularly had received greater attention due to their close proximities resulting in pre-activation prior to the limb movement and thus contributing to the core stability of the lumbopelvic region (3). For example, 20.9\% of reduced thickness and the delayed anticipatory onset of $\operatorname{Tr} A$ muscle during limb loading had been reported among patients with NSLBP $(4,5)$. Recently, the measure of $\operatorname{Tr} A$ morphometry had been 
suggested to be a treatment effect modifier for NSLBP patients (6). On the other hand, the thickness of TrA had been studied in terms of gender difference, between rest and contraction, hand dominance and side to side differences among healthy participants to understand the normative function of $\operatorname{TrA}$ muscle $(7,8)$. Therefore, many clinicians might consider the assessment and treatment of the core muscles as one of the factors towards the management of NSLBP (9). Although several pieces of evidence were available on TrA muscle anatomy and function, the changes in TrA muscle during the different levels of the LPST through an abdominal draw-in maneuver had never been studied before.

The function of the TrA was evaluated by clinicians through an abdominal drawing-in manoeuvre using a pressure biofeedback unit (PBU) $(10,11)$. In theory, the PBU was placed under the lumbar spine in crook lying position and the patients were instructed to tuck in the abdomen. Any pressure fluctuations greater than $4 \mathrm{mmHg}$ during a standard test using $40 \mathrm{mmHg}$ was considered as poor stability of the lumbar spine. If no fluctuations, the participants performed a unilateral leg lift in the sagittal plane followed by the seven progressive levels of LPST (12). An ability to maintain the registered pressure at 40 $\mathrm{mmHg}$ during the testing movement was marked as a successful performance. When the participants were not able to hold $40 \pm 4 \mathrm{mmHg}$ in the $\mathrm{PBU}$, the progression of the LPST would be stopped.

Although testing the TrA muscle using the PBU was reported to have good reliability of the TrA function (11), the concurrent validity was reported to be poor against the electromyography (13). A recent systematic review concluded that the ability of the test to directly assess the function of TrA had not been answered (14). Perhaps, this was due to the fact that the test did not measure directly the changes in the $\operatorname{Tr} A$, instead, it measured the changes in the pressure of the PBU as an indirect evaluation of the TrA muscle contractility. Till now, no studies had investigated the changes in the TrA function during different levels of the LPST as indicated by the different upper and lower limb movements. Furthermore, an absence of any normative understanding of the TrA muscle function among healthy individuals remains a challenge for clinicians to interpret its outcome among low back pain patients. Therefore, the main aim of the study is to investigate the changes in the contraction ratio of the TrA muscle during different levels of the LPST among healthy participants. The study hypothesizes that the contraction ratio of $\operatorname{Tr} A$ and IO differs significantly during different levels of the LPST. The current study is the first study to report on the contraction ratio of the TrA and IO during the different levels of the LPST. It is important to establish normative data in the healthy population as it becomes a benchmark for any conditions such as NSLBP.

\section{STUDY DESIGN}

A cross-sectional study with an experimental design was conducted to investigate the differences in $\operatorname{Tr} A$ and IO during different levels of the LPST. The details of the study were advertised through posters around a community setting. The participants for the study were recruited through pre-defined study criteria from a community population. The study was carried out in the pain and neuromusculoskeletal research laboratory in a university setting. The study was conducted according to the international ethical standards recommended in the clinical and field science research (15). A university institutional ethics board approved the ethics of the study according to the Declaration of Helsinki.

\section{PARTICIPANT CHARACTERISTICS}

A total of among a total of 30 healthy individuals $(15$ males and 15 females) participated in the study. Any participants who did not have body aches or pain over the last 3 months were invited to participate in the study. The participants were excluded if they had referred pain or neurological involvement in lower limbs, had any history of past surgery and had any history of injury in the last 3 months. The purpose of the study and the study procedure were explained to all the participants and written informed consent was obtained prior to their participation in the study.

\section{PROCEDURE}

\section{Lumbopelvic stability testing}

A pressure biofeedback stabilizer unit (PBU) (Stabilizer Pressure Biofeedback-Chattanooga Group, USA) was used during the test. PBU was reported to be a reliable device to test the lumbopelvic stability (16). The LPST was performed in supine crook lying position with the participant hip and knee flexed to $70^{\circ}$ and $90^{\circ}$ respectively through an established protocol (12). Before the test, the PBU was calibrated and pre-tested through an established protocol by loading the unit for 24 hours with $4 \mathrm{~kg}$ of weight (17). The PBU unit was considered for use only if the device lost no more than $0.4 \mathrm{~mm} \mathrm{Hg}$ during the testing period and the same unit was used throughout the study period. After calibration, the PBU was placed beneath the lumbar spine between the levels of the second lumbar spine and the first sacral spine. The participants were instructed to tuck in 
their naval by drawing in the abdomen. The pressure in the pressure gauge was maintained at $40 \mathrm{~mm} \mathrm{Hg}$ and the participants were instructed not to exceed more than $4 \mathrm{~mm}$ $\mathrm{Hg}$ during the abdominal drawing-in manoeuvre. Once the participants were able to maintain the pressure, the tests were progressed into 7 levels in the following order (12); core with alternate hip abduction, core with alternate knee raise, core with both arms adduction, core with both arms extension, core with alternate arm lift, and core with alternate leg lift and finally core with alternate leg and arm lift. The test was stopped when the participants exceeded the pressure more than $40 \pm 4 \mathrm{~mm} \mathrm{Hg}$ and the level of the test was noted as completed.

\section{Real-time ultrasound measurement of $\operatorname{Tr} A$}

The real-time ultrasound imaging (USI) measurement of the TrA muscle was carried using a B-mode real-time ultrasound imaging unit (TOSHIBA, Famio8, SSA-530A) through an established protocol (18). The ultrasound measurement was carried out simultaneously when the participants performed the different levels of the LPST. The participants were positioned in crook lying with their head and knee supported by pillows. Ultrasonic gel was applied to a $5-\mathrm{MHz}$ curvilinear transducer and the transducer was placed in the transverse plane over the midaxillary line at a midpoint between the lower rib and the iliac crest. An experienced physiotherapist who was trained to conduct USI measurement carried out all the measurements. A surface marking was made on the skin using a surface marker to ensure the transducer was placed over the same location for every measurement during the seven levels of the test.

The images of $\operatorname{Tr} A$ thickness were captured and measured using NIH (Bethesda, MD) Image J Software (Version 1.8). The total resting muscle thickness was defined as the distance between the superior border and the inferior border of TrA muscle. Then, the patients were instructed to draw in their abdomen and $\operatorname{Tr} A$ muscle thickness during contraction was measured during the 7 levels of the test. Figure 1 shows the USI measurement of the muscle thickness during the LPST. All the measurements were taken at the end of the expiration. The mean thickness of the three measurements on each side was calculated for analysis. The contraction ratio of the TrA muscle was calculated for all the 7 levels of the test using an established formula (19).

$$
\text { Contraction ratio }=\frac{\text { Muscle thickness during contraction }}{\text { resting muscle thickness }}
$$

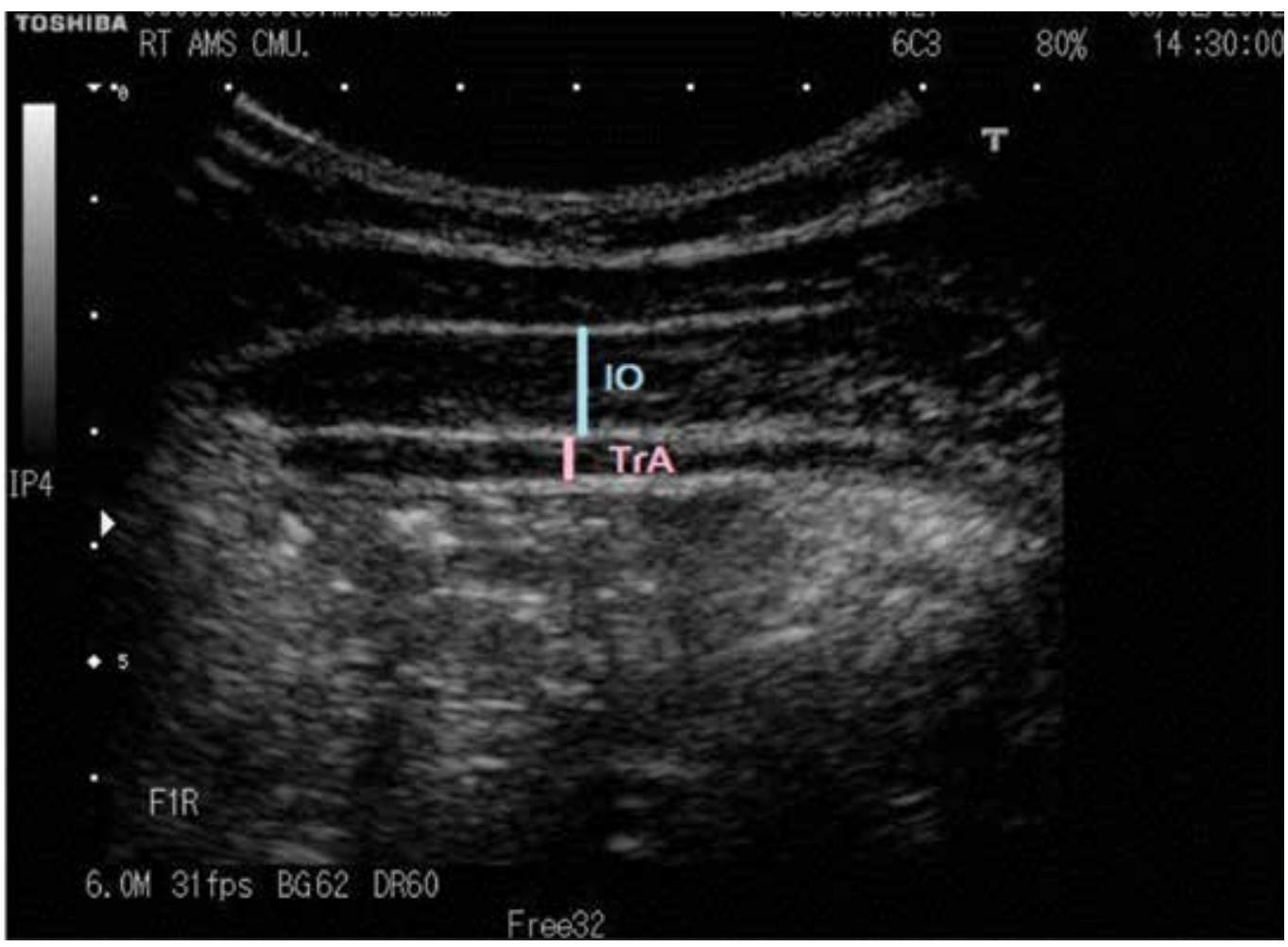

Figure 1. Ultrasound imaging of the internal oblique and transversus abdominis. 


\section{STATISTICAL ANALYSIS}

The sample size for the study was calculated using the $G^{*}$ Power software program by selecting 2-tails comparison between groups with an effect size of 0.71 for the primary outcome TrA with a power of $95 \%$, alpha error probability of 0.05 which suggested a sample size of at least 15 per gender. The statistical software package for social sciences (SPSs windows version 22.0) was used for data analysis (20). The measured values were normally distributed according to the Kolmogorov-Smirnov test. The differences in the contraction ratio of $\mathrm{Tr} A$ and IO muscles between the 7 levels of testing were analysed using One-way Repeated measures ANOVA. The level of significance was set as 0.05 . In terms of any significant differences, a Post hoc was analysed using Bonferroni at $\mathrm{p}<0.05$.

\section{RESULTS}

A total of 30 healthy individuals (15 males and 15 females) with a mean age of 21,83 years participated and completed the study. The descriptive characteristics of the study participants were given in table I. The mean (SD) value of the resting thickness and the thickness during contraction of $\operatorname{Tr} \mathrm{A}$ and IO for all the 7 levels of the LPST were shown in table II. The result showed that the values of the contraction ratio of the $\operatorname{Tr} A$ and IO decreased as the tests were progressed into the different 7 levels of the testing. The results of the post hoc analysis showed that the contraction

Table I. Characteristics of the study participants.

\begin{tabular}{llll}
\hline Variables & Mean \pm SD & Min & Max \\
\hline age (years) & $21.83 \pm 0,46$ & 20.00 & 22.00 \\
\hline weight $(\mathrm{kg})$ & $55.48 \pm 11.6$ & 41.00 & 100.00 \\
\hline height $(\mathrm{cm})$ & $163.87 \pm 8.14$ & 150.00 & 181.00 \\
\hline BMI $\left(\mathrm{kg} / \mathrm{m}^{2}\right)$ & $20.541 \pm 3.28$ & 16.61 & 35.43 \\
\hline
\end{tabular}

ratio of TrA significantly reduced in the 7 th levels of the testing $(\mathrm{F}=14.53, \mathrm{p}=0.001)$. However, the contraction ratio of IO remains unchanged in all the 7 levels of LPST.

\section{DISCUSSION}

This was the first study that investigated the changes in the contraction ratio of $\operatorname{Tr} A$ and IO muscles during the 7 different progressive stages of the LPST. The findings of the study suggested that $\operatorname{Tr} A$ muscle possibly were showing signs of fatigue as the muscle was repeatedly made to contract during the 7 progressive levels of the LPST. In clinical practice, one might argue that this was the characteristic pattern of the LPST which might explain that the difficulty levels of the 7 progressive stages of the test might demand an increasing workload on $\operatorname{Tr} A$ muscle. It was worth noting that a past study challenged the notion that the PBU could clinically assess TrA contraction during the abdominal drawing-in manoeuvre (10). However, the findings on $\operatorname{Tr} A$ muscle were proposed based on a population of NSLBP and the TrA muscle was not tested in different levels of LPST. In contrast, the current study might suggest that the abdominal drawing-in task through PBU had engaged TrA and the TrA muscle responded with decreasing values of contraction thickness across the 7 levels of the LPST. While the past studies used PBU to measure the lumbopelvic stability as an indirect measurement of $\operatorname{Tr} A$ muscle function $(10,11)$ none of the past studies directly measured how the TrA muscle adapts and functions to the 7 progressive stages of the LPST. Therefore, the current study findings might provide a direct measurement of the TrA with the normative value which could provide clinicians an understanding of how TrA muscle responds to the LPST on healthy individuals. An understanding of the normative values and function of TrA muscle might help clinicians to interpret the test results among NSLBP.

The average values of the contraction ratio of TrA muscle in the current study were similar to other established evidence.

Table II. Transversus abdominis and internal oblique muscles thickness and contraction ratio during 7 levels of lumbopelvic stability test (LPST).

\begin{tabular}{|c|c|c|c|c|c|c|c|c|c|}
\hline \multirow{2}{*}{ Variables } & \multirow{2}{*}{ Muscle } & \multicolumn{8}{|c|}{ LPST level } \\
\hline & & resting & $1^{\text {st }}$ & $2^{\text {nd }}$ & $3^{\text {rd }}$ & $4^{\text {th }}$ & $5^{\text {th }}$ & $6^{\text {th }}$ & $7^{\text {th }}$ \\
\hline \multirow{2}{*}{$\begin{array}{l}\text { Muscle } \\
\text { Thickness } \\
\text { (mm) }\end{array}$} & $\operatorname{Tr} A$ & $\begin{array}{c}2.23 \pm \\
0.5\end{array}$ & $4.07 \pm 0.9$ & $4.21 \pm 0.9$ & $4.10 \pm 1.1$ & $3.76 \pm 1.1$ & $3.85 \pm 1.2$ & $3.67 \pm 1.2$ & $3.17 \pm 1.2$ \\
\hline & $\mathrm{IO}$ & $\begin{array}{c}5.38 \pm \\
1.9 \\
\end{array}$ & $6.11 \pm 2.7$ & $6.10 \pm 2.4$ & $6.41 \pm 2.6$ & $7.09 \pm 5.4$ & $6.21 \pm 2.3$ & $6.24 \pm 2.5$ & $6.22 \pm 2.2$ \\
\hline $\begin{array}{l}\text { Contraction } \\
\text { Ratio }\end{array}$ & $\operatorname{Tr} A$ & - & $1.89 \pm 0.4$ & $1.95 \pm 0.4$ & $1.88 \pm 0.4$ & $1.73 \pm 0.4$ & $1.76 \pm 0.5$ & $1.70 \pm 0.5$ & $1.46 \pm 0.5^{*}$ \\
\hline
\end{tabular}

*significantly difference at $\mathrm{p}<0.001$ when compared to $1^{\text {st }}$ level of LPST 
For instance, the range of $\operatorname{Tr} A$ contraction ratio measured in our study ranged between $1.5 \mathrm{~mm} \mathrm{Hg}$ and $1.8 \mathrm{~mm} \mathrm{Hg}$ which fell within the range of $1.5 \mathrm{~mm} \mathrm{Hg}$ up to $2.2 \mathrm{~mm} \mathrm{Hg}$ $(21,22)$. It could be suggested that the norms of measurements were acceptable within the practice standards. The contraction ratio of IO had been reported to be the thinnest during rest and thickest during the abdominal drawing-in manoeuvre (23). Also, irrespective of the measurements of the cross-section of $\operatorname{Tr} \mathrm{A}$ and IO either at rest or during the testing procedure, it was reported that $\mathrm{IO}$ and $\operatorname{Tr} A$ muscles remained the thickest and the thinnest, respectively (23). However, the cross-section ratio of $\operatorname{Tr} A$ was the thickest ranging from 1.5-1.8 $\mathrm{mm} \mathrm{Hg}$, while the cross-section ratio of the IO was the thinnest ranging from 1.1-1.3 $\mathrm{mm} \mathrm{Hg}$. Perhaps, the difference could be because the previous study tested the contraction ratio of $\operatorname{Tr} \mathrm{A}$ and IO during the abdominal drawing-in task, while the current study used a tougher testing procedure of testing the muscles across 7 levels of the LPST. Also, the past study reported values from the low back pain population, while the findings of the current study were from healthy individuals. Furthermore, the current findings were in line with a recently reported evidence among healthy participants that the activation levels of the $\operatorname{Tr} A$ were between 1.5-2.0 mm $\mathrm{Hg}(24,25)$. Therefore, it could be possible that the cross-section ratio of TrA and IO muscles might adapt and respond differently between healthy individuals and NSLBP population. Therefore, further studies were warranted to test the adaptation of the cross-section ratio of $\operatorname{Tr} \mathrm{A}$ and IO muscles across the 7 levels of LPST between healthy individuals and NSLBP.

\section{CLINICAL IMPLICATION}

The current study might have some practice implications. Reference values of the clinical tests among healthy individuals are necessary, as these results may contribute to clinical

\section{REFERENCES}

1. Panjabi MM. A hypothesis of chronic back pain: ligament sub failure injuries lead to muscle control dysfunction. Eur Spine J, 2006, 15 (5): 668-676.

2. P.W. Hodges, G.L. Moseley. Pain and motor control of the lumbopelvic region: effect and possible mechanisms. J Electromyogr Kinesiol, 2003. 13 (4): 361-370.

3. Hodges PW. Spinal segmental stabilization training in rehabilitation of the spine. Lippincott: Williams \& Wilkins; 2007.

4. Teyhen DS, Bluemle LN, Dolbeer JA, Baker SE, Molloy JM, Whittaker J, Childs JD. Changes in lateral abdominal muscle thickness during the abdominal drawing-in maneuver in those with lumbopelvic pain. J Orthop Sports Phys Ther, 2009, 39(11):791-8. practice and for setting up training targets (26). For instance, an understanding of certain baseline normative data on the contraction ratio of $\mathrm{Tr} A$ and $\mathrm{IO}$ among the healthy individuals during the LPST might provide clinicians a reference point and comparison standard for NSLBP. A past study raised a clinical question about the measurement property of PBU to engage $\operatorname{Tr} A$ (15). Also, the engagement of $\operatorname{Tr} A$ with $\mathrm{PBU}$ was questioned due to poor correlation with electromyography (27). In the above context, the current study findings supported that $\operatorname{Tr} \mathrm{A}$ and IO were engaged and activated using a PBU and USI supporting the notion that the contraction ratio of $\operatorname{Tr} A$ muscle could be evaluated using the 7 levels of the LPST. The small sample size is one of the limitation for the study. Although a sample size of 15 participants were identified as sufficient for a gender in the study, one might suggest that the study consisted of a small number of research participants and hence, the findings might be more prone to variability. Also, another limitation of this study might be mixing the results of men and women, as the thickness of the muscles might be associated with gender.

\section{CONCLUSIONS}

A significant reduction in the contraction ratio of $\operatorname{Tr} A$ was observed in the $7^{\text {th }}$ level of the LPST as measured by the PBU and USI. However, the contraction ratio of IO remains unchanged during all the 7 levels of LPST. As the levels of the LPST increased, the contraction ratio of deep stabilizing muscles was insufficient to stabilize the lumbopelvic region. Further studies are warranted to compare the adaptation of $\mathrm{Tr} \mathrm{A}$ and IO muscles across 7 levels of the testing among patients with NSLBP.

\section{CONFLICT OF INTERESTS}

The authors declare they have no conflict of interests.

5. MacDonald D, Moseley GL, Hodges PW. Why do some patients keep hurting their back? Evidence of ongoing back muscle dysfunction during remission from recurrent back pain. $P A I N^{\circledR}, 2009,142: 183-188$.

6. Unsgaard-Tфndel M, Nilsen TIL, Magnussen J, Vasseljen O. Is activation of transversus abdominis and obliquus internus abdominis associated with long-term changes in chronic low back pain? A prospective study with 1-year follow-up. BM] Sports Med, 2012, 46: 729-734.

7. Springer BA, Mielcarek BJ, Nesfield TK, Teyhen DS. Relationships among lateral abdominal muscles, gender, body mass index, and hand dominance. J Orthop Sports Phys Ther, 2006 , 36(5): 289-97. 
8. Rho M, Spitznagle T, Van Dillen L, Maheswari V, Oza S, Prather H. Gender differences on ultrasound imaging of lateral abdominal muscle thickness in asymptomatic adults: a pilot study. PM R, 2013, 5(5): 374-80.

9. Şahin N, Karahan AY, Albayrak I. Effectiveness of physical therapy and exercise on pain and functional status in patients with chronic low back pain: a randomized controlled trial. Turk J Phys Med Rehab 2018;64(1):52-58.

10. Grooms DR, Grindstaff TL, Croy T, Hart JM, Saliba SA. Clinimetric analysis of pressure biofeedback and transversus abdominis function in individuals with stabilization classification low back pain. J Orthop Sports Phys Ther, 2013, 43(3): 184-93.

11. Lima PO, de Oliveira RR, de Moura Filho AG, Raposo MC, Costa LO, Laurentino GE. Reproducibility of the pressure biofeedback unit in measuring transversus abdominis muscle activity in patients with chronic nonspecific low back pain. J Bodyw Mov Ther, 2012, 16(2): 251-7.

12. Paungmali A, Henry LJ, Sitilertpisan P, Pirunsan U, Uthaikhup S. Improvements in tissue blood flow and lumbopelvic stability after lumbopelvic core stabilization training in patients with chronic non-specific low back pain. J Phys Ther Sci, 2016, 28(2): 635-40.

13. Lima PO, Oliveira RR, Moura Filho AG, Raposo MC, Costa LO, Laurentino GE. Concurrent validity of the pressure biofeedback unit and surface electromyography in measuring transversus abdominis muscle activity in patients with chronic nonspecific low back pain. Rev Bras Fisioter, 2012, 16(5): 389-95.

14. De Paula Lima PO, de Oliveira RR, Costa LO, Laurentino GE. Measurement properties of the pressure biofeedback unit in the evaluation of transversus abdominis muscle activity: a systematic review. Physiotherapy, 2011, 97(2): 100-6.

15. Padulo J., Oliva F., Frizziero A., Maffulli N. Muscles, Ligaments and Tendons Journal - Basic principles and recommendations in clinical and field Science Research: 2018 update. MLTJ 2018; 8(3): 305-307.

16. Azevedo DC, Lauria AC, Pereira ARS, Andrade GT, Ferreira ML, Ferreira PH, Van Dillen L. Intraexaminer and interexaminer reliability of pressure biofeedback unit for assessing lumbopelvic stability during 6 lower limb movement test. J Manipulative Physiol Ther 2013; 36:33-43.

17. K von Garnier, K Koveker, B Rackwitz, U Kober, S Wilke, T Ewert, et al.Reliability of a test measuring transversus abdomi- nis muscle recruitment with a pressure biofeedback unit. Physiotherapy, 2009, 95: 8-14.

18. Sitilertpisan P, Pirunsan U, Puangmali A, Ratanapinunchai J, Kiatwattanacharoen S, Neamin H, Laskin JJ. Comparison of lateral abdominal muscle thickness between weightlifters and matched controls. Phys Ther Sport, 2011, 12: 171-4.

19. Pulkovski N, Mannion AF, Caporaso F, Toma V, Gubler D, Helbling D, Sprott H. Ultrasound assessment of transversus abdominis muscle contraction ratio during abdominal hollowing: a useful tool to distinguish between patients with chronic low back pain and healthy controls? Eur Spine J. 2012;21 (Suppl 6): S750-S759.

20. IBM Corp. Released 2011. IBM SPSS Statistics for Windows, Version 22.0. Armonk, NY: IBM Corp.

21. Linek P, Saulicz E, Wolny T, Myśliwiec A, Kokosz M. Lateral abdominal muscle size at rest and during abdominal drawing-in manoeuvre in healthy adolescents. Man Ther, 2015, 20(1): 117-23.

22. F.D. Manshadi, M. Parnianpour, J. Sarrafzadeh, M.R. Azghani, A. Kazemnejad. Abdominal hollowing and lateral abdominal wall muscles' activity in both healthy men \& women: an ultrasonic assessment in supine and standing positions. J Bodyw Mov Ther, 2011, 15: 108-113.

23. Mannion et al., 2008b, A.F. Mannion, N. Pulkovski, V. Toma, H. Sprott. Abdominal muscle size and symmetry at rest and during abdominal hollowing exercises in healthy control subjects. J Anat, 2008, 213: 173-182.

24. Teyhen DS, Rieger JL, Westrick RB, Miller AC, Molloy JM, Childs JD. Changes in deep abdominal muscle thickness during common trunk-strengthening exercises using ultrasound imaging. J Orthop Sports Phys Ther, 2008, 38: 596605.

25. Gorbet N, Selkow NM, Hart JM, Saliba S. No Difference in Transverse Abdominis Activation Ratio between Healthy and Asymptomatic Low Back Pain Patients during Therapeutic Exercise. Rehabil Res Pract. 2010; 2010:459738.

26. Oliveira IO, Pilz B, Santos RLG Junior, Vasconcelos RA, Mello W, Grossi DB. Reference values and reliability for lumbopelvic strength and endurance in asymptomatic subjects. Braz J Phys Ther, 2018, 22(1): 33-41.

27. O.M. Giggins, U.M. Persson, B. Caulfield. Biofeedback in rehabilitation. J Neuroeng Rehabil, 2013, 10: 60. 